\title{
Johnson-Nyquist noise and the Casimir force between real metals at nonzero temperature
}

\author{
Giuseppe Bimonte \\ Dipartimento di Scienze Fisiche Università di Napoli Federico II \\ Complesso Universitario MSA Via Cintia I-80126 Napoli Italy; \\ INFN, Sezione di Napoli, Napoli, ITALY \\ E-mail: Bimonte@na.infn.it
}

\begin{abstract}
It is well known since a long time that all lossy conductors at finite temperature display an electronic noise, the Johnson-Nyquist noise, arising from the thermal agitation of electric charges inside the conductor. The existence of this noise implies that two nearby discharged conductors at finite temperature should repel each other, as a result of the electrodynamic interaction between the Johnson-Nyquist currents in either conductor and the eddy currents they induce in the other. It is suggested that this force is at the origin of the recently discovered large repulsive correction to the thermal Casimir force between two lossy metallic plates. Further support for this physical picture is obtained by studying a simple system of two linear noisy antennas. Using elementary concepts from circuit theory, we show that the repulsive force engendered by the Johnson-Nyquist noise results in the same kind of thermodynamic inconsistencies found in the Casimir problem. We show that all inconsistencies are however resolved if account is taken of capacitive effects associated with the end points of the antennas. Our findings therefore suggest that capacitive effects resulting from the finite size of the plates, may be essential for a resolution of the analogous problems met in the thermal Casimir effect.

PACS numbers: 05.40.-a, 42.50.Lc,74.45.+c
\end{abstract}

\section{Introduction}

In recent years, great advances in experimental techniques have stimulated an intense theoretical and experimental activity on the Casimir effect, and more in general on dispersion forces. For recent reviews on this rapidly evolving field, we address the reader to Refs.[1]. The present contribution reports on our recent work [2] on a theoretical conundrum that attracted much attention in the last few years, and is not yet resolved as we write. The problem is that of determining the influence of temperature on the Casimir force between two conductors, when the latter are treated as real metals, i.e. taking account of ohmic losses. This is not an academic question, because the increasing accuracy of Casimir force measurements is rapidly approaching the point, where these thermal corrections should become measurable. In fact, in a related context, the effect of temperature on the Casimir-Polder interaction between a Bose-Einstein condensate 
Johnson-Nyquist noise and the Casimir force between real metals at nonzero temperature 2

and a dielectric surface has been recently observed [3]. The problem of the influence of temperature on the Casimir force is an old one, for it was already discussed in some detail, both for dielectrics and conductors, by Lifshitz in his seminal paper on the theory of molecular attractive forces between solids [4]. As its is well known, in this macroscopic theory the bodies are characterized by means of a dielectric permittivity $\epsilon(\omega)$ (we shall not consider in what follows magnetic materials). When dealing with conductors, Lifshitz pointed out that for plate separations $a$ of the order of one micron, the important region of frequencies is $\omega / c \simeq 1 / a$, corresponding to the infrared part of the spectrum. At such high frequencies ohmic losses are irrelevant, and accordingly Lifshitz took for the dielectric function of the metal the expression appropriate in the infrared, i.e. the plasma model $\epsilon_{P}(\omega)$

$$
\epsilon_{P}(\omega)=1-\Omega_{p}^{2} / \omega^{2},
$$

where $\Omega_{p}$ is the plasma frequency. Lifshitz used it to estimate the leading temperature correction to the Casimir pressure. He observed in passing that for larger separations, i.e. for lower frequencies, the plasma dielectric function should be replaced by

$$
\epsilon=4 \pi i \sigma / \omega,
$$

where $\sigma$ is the ordinary electrical conductivity of the metal, but no explicit computations were performed for this case. After many years, the question was revived in Ref. [5], which contains the first explicit computation of the thermal correction to the Casimir pressure, taking account of dissipation. For this purpose, when evaluating Lifshitz formula giving the Casimir pressure between two infinite plates at finite temperature, the authors of [5] used the well-known Drude model

$$
\epsilon_{D}(\omega)=1-\frac{\Omega_{p}^{2}}{\omega(\omega+i \gamma)},
$$

which provides a smooth interpolation between Eq. (1) and Eq. (2) (the relaxation frequency $\gamma$ in Eq. (3) is related the conductivity as $\gamma=\Omega_{p}^{2} /(4 \pi \sigma)$ ). Replacement of Eq. (1) by Eq. (3) in Lifshitz formula produced an unexpected result because, in the case of good conductors, instead of the expected small correction, Lifshitz formula predicted a large repulsive correction, to the extent that for large separations the Casimir force was halved with respect to the predicted result for the model without dissipation. In fact, the magnitude of this repulsive correction is independent of the actual magnitude of the dissipation, provided that some dissipation is present. The mathematical reason for this result is clear [5], because Lifshitz formula for the Casimir pressure at finite temperature consists of a sum over imaginary discrete frequencies $\xi_{n}=2 \pi n k_{B} T / \hbar, n=0,1, \ldots$ (the so-called Matsubara frequencies). Its first term, for $n=0$, provides the leading contribution at large temperature and/or separations and since it involves the quantity $A=\lim _{\xi \rightarrow 0} \epsilon(i \xi) \xi^{2}$, the magnitude of this term depends crucially on the behavior of the dielectric function at zero frequency. Even for infinitesimal amounts $\gamma$ of dissipation in Eq. (3), the quantity $A$ jumps discontinuously from the plasma-model value $\Omega_{p}^{2}$ to zero, resulting in a discontinuous change in the thermal correction to the Casimir pressure, as soon as dissipation is turned on. 
Johnson-Nyquist noise and the Casimir force between real metals at nonzero temperature 3

Another problem was later pointed out in [6], where it was found that for metallic plates without impurities, straightforward insertion of the Drude model into Lifshitz formula results in a violation of the Nernst heat theorem, while no such inconsistencies arise if the plasma model Eq. (11) is used. Analogous thermodynamical inconsistencies have also been found in the case of dielectrics, when a Drude-like term is added to the permittivity function to account for their small dc electric conductivity [7]. These puzzling results stimulated a lively debate among the experts, and several different attitudes have emerged (for a survey, see Refs. [8, 9] and the contribution to these Proceedings by V. M. Mostepanenko). At the moment of this writing, the experimental situation is not yet clear. Recent measurements of the Casimir force in the submicron range [10, where thermal effects are small anyhow, appear to exclude the repulsive thermal correction implied by the Drude model and seem to be in agreement with the plasma model prediction, but the actual level of precision achieved by these experiments has been questioned by some authors [9]. Very likely, for a definitive experimental resolution of the problem, we shall have to wait until the measurements will be extended to separations of a few microns, for which rival theoretical approaches give largely different predictions for the thermal Casimir force.

\section{The physical origin of the large thermal correction}

In what follows, we shall argue that the jump in the thermal Casimir force predicted by Lifshitz formula, is not a mere mathematical accident but it rather signals that, when dissipation is turned on, a new physical phenomenon, specifically connected with dissipation in conductors, is sneaking into the problem of the thermal Casimir force. In our view, discovering if such a new phenomenon is actually present is of great importance, because this might help us understanding if and when Lifshitz theory can be used to describe it.

An important hint is obtained from Refs. [11], where the problem was investigated using an equivalent mathematical formulation of Lifshitz theory, that allows a clean separation of the contribution of thermal photons from that of zero-point fluctuations. In this way, it was shown that dissipation has a small influence on the contribution from zero-point fluctuations, and that the large thermal repulsive correction found in [5] originates from thermally excited evanescent photons, with transverse (TE) polarization. It is important to note that no such waves exist when dissipation is absent, as it occurs when the plasma model is used instead of the Drude model. The important (real) frequencies of these fluctuations are of order $\tilde{\omega}=\gamma\left(\omega_{c} / \Omega_{p}\right)^{2}$, where $\omega_{c}=c / a$ is the characteristic frequency of the cavity [16]. For typical Casimir experiments, and even more so at low temperature, when $\gamma$ is small, $\tilde{\omega}$ is much less than $\omega_{c}$ and therefore these near fields have nothing to do with the cavity eigen-modes whose zero-point energies are at the origin of the Casimir effect proper. In fact, since typically $\tilde{\omega} \ll k_{B} T / \hbar$ the repulsive force associated with these TE evanescent fields is a classical effect [16].

We can ask what produces these fields. At the low frequencies involved retardation 
Johnson-Nyquist noise and the Casimir force between real metals at nonzero temperature4

effects are negligible and the relevant $\mathrm{TE}$ evanescent fields represent quasi-static magnetic fields. It is natural to imagine that the source of these magnetic fields are thermally excited electric currents inside the plates, and one is immediately led to identify them with the currents that are at the origin of the familiar Johnson-Nyquist noise 12. As it is well known, this is the electronic noise characteristic of resistive conductors at finite temperature, originating from the thermal agitation of charges inside a conductor. A striking feature of this noise is that it exists only in conductors that display some electrical resistance, and it is absent in strictly dissipationless conductors, explaining why we do not see it if we use for the metal a model with no dissipation, like the plasma model in Eq. (11). The repulsive correction to the thermal Casimir force found in [5] appears now as the natural effect of the electrodynamic interaction between the Johnson currents in either plate and the eddy currents induced in the other plate. From this new perspective, the whole question appears basically as a slightly unconventional problem in electrical engineering, i.e. the problem of the mechanical interaction between two nearby noisy (extended) antennas. In order to put to a test these ideas, in the next Section we shall investigate the mechanical force existing between two linear noisy antennas. Since two wires do not form a cavity, by considering this simple system we get rid of the spurious complication added by the eigen-modes of a Casimir apparatus, and we can isolate the features of the interaction caused by the Johnson-Nyquist noise.

\section{Mechanical interaction between two noisy linear antennas}

Let us consider a system of two identical discharged linear antennas, consisting of two pieces $\mathcal{C}_{1}$ and $\mathcal{C}_{2}$ of thin metallic wire at temperature $T$, displaced by an amount $\vec{a}$ from each other (we consider the orientations of the wires as fixed once and for all). We assume that the thickness of the wires is much smaller than the separation $a$, and that their length $L$ is small compared with the wavelength $\lambda$ of the radiated e.m. fields, such that we can describe the two antennas as two concentrated resistors with resistance $R$ (depending solely on the temperature). As it is well known [12], the Johnson-Nyquist noise in a noisy resistor can be described by replacing the actual resistors by noiseless ones, connected each to a noise generator producing a random e.m.f. $\mathcal{E}_{i}(t), i=1,2$ with spectrum:

$$
\left\langle\mathcal{E}_{i}(\omega) \mathcal{E}_{j}^{*}\left(\omega^{\prime}\right)\right\rangle=4 \pi k_{B} T R E\left(\omega / \omega_{T}\right) \delta\left(\omega-\omega^{\prime}\right) \delta_{i j},
$$

where angle brackets denote statistical averages, $i, j=1,2, \omega_{T}=k_{B} T / \hbar$ and $E(y)=$ $y\left(e^{y}-1\right)^{-1}$.

Obviously, besides a resistance, the two antennas are characterized by a selfinductance $\mathcal{L}$, and a mutual inductance $\mathcal{M}(\vec{a})$ depending on the separation $\vec{a}$. For the moment we shall not consider that the antennas, having a finite length, possess

also capacitances $C$ associated with their end-points. One might say that neglecting these edge-effects is correct, as far as $a \ll L$, analogously to what is always done in the 
Johnson-Nyquist noise and the Casimir force between real metals at nonzero temperature5

Casimir problem, where the plates are considered to be infinite, as in Lifshitz theory, because one assumes that for plate separations much smaller than the plates size, edge effects are negligible. The system of two antennas is then described by the following circuit equations:

$$
\begin{aligned}
& \mathcal{L} \frac{d i_{1}}{d t}+\mathcal{M}(\vec{a}) \frac{d i_{2}}{d t}+R i_{1}=\mathcal{E}_{1}(t), \\
& \mathcal{L} \frac{d i_{2}}{d t}+\mathcal{M}(\vec{a}) \frac{d i_{1}}{d t}+R i_{2}=\mathcal{E}_{2}(t) .
\end{aligned}
$$

From classical electrodynamics [13], one learns that the force $\vec{F}(\vec{a})$ between two inductances can be written as:

$$
\vec{F}(\vec{a})=\left\langle i_{1} i_{2}\right\rangle \vec{\nabla}_{a} \mathcal{M}(\vec{a}) .
$$

The correlator $\left\langle i_{1} i_{2}\right\rangle$ can be easily computed by taking the time-Fourier transform of Eqs.(5) and using Eq. (44). After some computations, we obtain for the force the simple formula:

$$
\vec{F}=-k_{B} T H \vec{\nabla}_{a}\left(m^{2}\right),
$$

where $m=\mathcal{M} / \mathcal{L}$ and $H$ is the quantity:

$$
H\left(\omega_{R} / \omega_{T}, m^{2}\right)=\frac{1}{\pi} \int_{0}^{\infty} d x x E\left(x \frac{\omega_{R}}{\omega_{T}}\right) \operatorname{Im}\left[(1-i x)^{2}+x^{2} m^{2}\right]^{-1},
$$

where $x=\omega / \omega_{R}$, with $\omega_{R}=R / \mathcal{L}$ (It should be noted that the frequencies $\omega$ that contribute to $H$ are in the range $0<\omega<\min \left\{\omega_{R}, \omega_{T}\right\}$, and therefore the long wavelength approximation used to write Eqs. (5) is justified, provided either $\omega_{R}$ or $\omega_{T}$ are small compared to $\left.c / L\right)$.

It is easy to verify that $H$ is positive definite, and therefore, since $\mathcal{M}^{2}$ decreases as $a$ increases, the force is repulsive. Moreover, since for small $R$ 's $E$ in Eq. (8) becomes one, and then $H$ becomes independent of $R$, it follows that in the limit of zero resistance the force attains the non-zero value

$$
\lim _{R \rightarrow 0} \vec{F}=-k_{B} T H\left(0, m^{2}\right) \vec{\nabla}_{a}\left(m^{2}\right) .
$$

However, it should be noted that the force is zero if we consider strictly dissipationless resistors. Consider now the free energy $\mathcal{F}$ associated with the force $\vec{F}$. Since $\vec{F}=-\vec{\nabla}_{a} \mathcal{F}$, we obtain from Eq.(7) and Eq.(8)

$$
\mathcal{F}=\frac{k_{B} T}{\pi} \int_{0}^{\infty} \frac{d x}{x} E\left(x \frac{\omega_{R}}{\omega_{T}}\right) \operatorname{Im} \log \left[1+\left(\frac{x m}{1-i x}\right)^{2}\right] .
$$

If the wires have no impurities, at liquid Helium temperatures and below, the resistance $R(T)$ approaches zero as $T^{2}$ [14]. Therefore for $T \rightarrow 0$, the ratio $\omega_{R} / \omega_{T}$ approaches zero, the integrand in Eq. (10) becomes independent of $T$ and we find that the free energy is of the form

$$
\mathcal{F} \approx g\left(m^{2}\right) k_{B} T
$$


Johnson-Nyquist noise and the Casimir force between real metals at nonzero temperature6

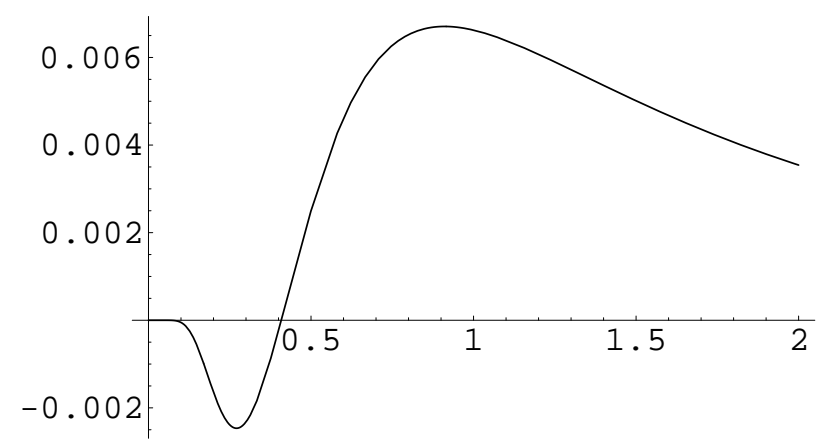

Figure 1. Plot of the free energy (in units of $\hbar \omega_{C}$ ) as a function of $t=k_{B} T /\left(\hbar \omega_{C}\right)$. See text for details.

where $g(z)$ is a positive function (because the imaginary part of the argument of the logarithm in Eq. (10) is positive definite). Recalling that the entropy $S$ is $S=-\partial \mathcal{F} / \partial T$, we then find:

$$
\lim _{T \rightarrow 0} S=-k_{B} g\left(m^{2}\right) \equiv S_{0}<0 .
$$

Since $S_{0}$ depends on the separation among the wires through $m^{2}$, this result represents a violation of Nernst heat theorem.

Summarizing, we have found that the Johnson-Nyquist noise in the antennas gives rise to a repulsive force, that does not vanish for vanishing resistance, is zero for strictly zero resistance and violates the Nernst heat theorem in the case of resistors with no impurities. These are exactly the same features displayed by the large repulsive thermal correction to the Casimir force between lossy plates, described in the Introduction. This analogy strongly supports the idea that the physical phenomenon behind this correction to the Casimir force is actually the Johnson noise in the plates.

\section{The role of the edges}

Given for granted that the Johnson-Nyquist noise exists and that it should give rise to a repulsion between two nearby conductors, the question arises whether the puzzling features of the force obtained in the previous Section are the consequence of some incorrect assumption. One of the assumptions that we made at the beginning of the previous Section is in fact suspicious, when we said that for small separations it is correct to neglect the capacitances associated with the end-points of the antennas. It is worth then to repeat the computations, taking full account of these capacitances, and see what happens. This is easy to accomplish, for all we have to do is to include the capacitances of the antennas in the circuit equations, Eq. (5). The resulting equations can be easily solved and one finds that, independently of how large the capacitances are, the results obtained in the previous Section change drastically in some important respects. Skipping all details, that can be found in Ref. [2], we just list the main points. 
First, the force now vanishes in the zero resistance limit, and thus one recovers smoothly the ideal metal result. The effect of the capacitances on the magnitude of the force for finite values of the resistance, depends of course on the value of the capacitance, and will not discussed here. The most remarkable change is however seen at low temperature, for instead of Eq. (11) we obtain now

$$
\mathcal{F}=-\frac{16 \pi^{5} m^{2}}{63}\left(\frac{k_{B} T}{\hbar \omega_{C}}\right)^{6} \hbar \omega_{R},
$$

where $\omega_{C}=1 / \sqrt{\mathcal{L} C}$. Obviously, the entropy is now positive, and vanishes as $T \rightarrow 0$, as required by Nernst theorem. However, numerical computations show that the entropy is negative at intermediate temperatures. This can be seen from the plot of the free energy (in units of $\left.\hbar \omega_{C}\right)$ as a function of the reduced temperature $t=k_{B} T /\left(\hbar \omega_{C}\right)$ in Fig.1. The curve was computed by taking $m=0.8$ and $\omega_{R}(t)=5 t^{2} \omega_{C}$. As we see, there is a region of temperatures $t$ where the slope of the curve is positive, which corresponds to a negative entropy. This is not necessarily a problem, though, because what needs to be positive is the total entropy of the system, which includes the self-entropies of the wires. Each wire, being now an $R L C$ oscillator, has a free energy $\mathcal{F}_{\text {self }}$ equal to:

$$
\mathcal{F}_{\text {self }}=k_{B} T \log \left[1-\exp \left(-\hbar \omega_{C} /\left(k_{B} T\right)\right)\right] .
$$

As we have checked numerically, inclusion of the wires self-entropies makes the total entropy of the system positive at all temperatures (we could not obtain an analytical proof of this). The important conclusion to be drawn is that inclusion of the antennas capacitances, however large they are, is sufficient to resolve all thermodynamical inconsistencies discovered in the previous Section.

\section{Conclusions}

In recent years much attention has been devoted to the study of the thermal Casimir force between lossy metallic plates. Interest in this problem arose as it was realized that even an infinitesimal amount of dissipation is sufficient to produce a large repulsive correction to the thermal Casimir force. In this paper, we advocate the view that the abrupt change in the thermal Casimir force indicates that dissipation brings into the problem a new physical phenomenon, characteristic of lossy conductors. We argued that this phenomenon is indeed the well known Johnson-Nyquist noise that exists in all conductors at finite temperature. From this point of view, the repulsive correction to the Casimir force can be simply explained as the electrodynamic interaction between the Johnson-Nyquist currents in either plate and the eddy currents they induce in the other plate. To verify this physical picture, we studied the electrodynamic forces arising between two linear noisy antennas. As a first guess, we solved the problem neglecting the capacitance of the antennas associated with their end-points, under the reasonable assumption that for separations between the antennas much smaller than the antennas lengths, edge effects would be negligible. This kind of assumption is customary in treatments of the Casimir effect, where standard Lifshitz theory assumes plates with 
infinite extension. After doing this approximation, we found a repulsive force that shares all the features found in the Casimir problem, in that it persists in the zeroresistance limit, and it violates the Nernst heat theorem for antennas without defects. These results appear to us as a clear indication of the fact that the Johnson-Nyquist noise is the physical phenomenon behind the large thermal correction to the Casimir effect for lossy plates.

In the antennas model we found that inclusion of the antennas capacitances, however large, drastically modifies the result, as they make the force vanish in the zero resistance limit and remove all thermodynamic inconsistencies as well. Whether capacitive edge effects are capable of resolving the analogous difficulties met in the thermal Casimir effect requires further investigations. In this connection, we remark that recently some authors have questioned the compatibility of the existence of real Johnson-Nyquist currents with Lifshitz theory, for the case of finite plates of any size [15]. In our view, capacitive edge-effects should be important only if the JohnsonNyquist current-fluctuations have a spatial size comparable to the plates size $L$. Detailed investigations of the thermal corrections to the Casimir force [11, 16] show that, at room temperature, the important current fluctuations are transverse and have characteristic frequency of order $\tilde{\omega}=\gamma\left(\omega_{c} / \Omega_{p}\right)^{2}$, and characteristic spatial size of the order of the plate separation $a$. Therefore, we expect that capacitive finite-size effects will be important, for plate separations $a$ not too much smaller than the plates size $L$. While this is not the case in standard Casimir experiments, this is indeed the typical situation in micro-mechanical devices, that are currently under intense investigation (see the third of Refs. [1]). At low temperature, the situation is more hopeful. As the temperature is lowered in the cryogenic range, the increasing degree of spatial correlation between the Johnson currents, implied by the anomalous skin effect [17, leads to a gradual suppression of fluctuations at small scales. It is conceivable that at very low temperatures, independently of the separation $a$, the current fluctuations become so correlated as to have a spatial extent comparable with the size of the plates. When this point is reached, edge effects enter into play and may become essential for a correct description, as discussed in this paper.

\section{References}

[1] Bordag M, Mohideen U and Mostepanenko V M 2001 Phys. Rep. 3531

Milton K A 2004 J. Phys. A 37 R209

Parsegian V A 2005 Van der Waals Forces (Cambridge University Press, Cambridge, England)

Capasso F, Munday J N, Iannuzzi D and Chan H B 2007 IEEE J. Sel. Top. Quant. Electron. 13 400

[2] Bimonte G 2007 New J. Phys. 9281

[3] Obrecht J M, Wild R J, Antezza M, Pitaevskii L P, Stringari S and Cornell E Q 2007 Phys. Rev. Lett. 98063201

[4] Lifshitz E M 1956 Sov. Phys. JETP 273

Lifshitz E M and Pitaevskii L P 1980 Landau and Lifshitz Course of Theoretical Physics: Statistical Physics Part II (Butterworth-Heinemann) 
Johnson-Nyquist noise and the Casimir force between real metals at nonzero temperature 9

[5] Bostrom M and Sernelius B E 2000 Phys. Rev. Lett. 844757

[6] Bezerra V B, Klimchitskaya G L and Mostepanenko V M 2002 Phys. Rev. A 65052113

Bezerra V B, Klimchitskaya G L, Mostepanenko V M and Romero C 2004 Phys. Rev. A 69022119

[7] Geyer B, Klimchitskaya G L and Mostepanenko V M 2005 Phys. Rev. D 72085009

[8] Klimchitskaya G L and Mostepanenko V M 2006 Contemp. Phys. 47131

[9] Brevik I, Ellingsen L A and Milton K A 2006 New J. Phys. 8236

[10] Decca R S et al. 2005 Ann. Phys. 31837

Decca R S et al 2007 Phys. Rev. D 75077101

Decca R S et al. 2007 Eur. Phys. J. C 51963

[11] Torgerson J R and Lamoreaux S K 2004 Phys. Rev. E 70047102

Lamoreaux S K 2005 Rep. Prog. Phys. 68201

Bimonte G 2006 Phys. Rev. E 73048101

[12] Johnson J B 1928 Phys. Rev. 3297

Nyquist H 1928 Phys. Rev. 32110

[13] Jackson J D 1999 Classical Electrodynamics (New York: Wiley)

[14] Kittel C 1996 Introduction to Solid State Physics (New York: Wiley).

[15] Geyer B, Klimchitskaya G L and Mostepanenko V M 2007 J. Phys. A: Math. Theor. 4013485

[16] Svetovoy V B and Esquivel R 2006 J. Phys. A: Math. Gen. 396777

[17] Svetovoy V B and Esquivel R 2005 Phys. Rev. E 72036113 